\title{
A CONSULTATIONS TRACKING DATABASE SYSTEM FOR IMPROVING FACULTY DEVELOPMENT CONSULTATION SERVICES
}

\author{
Jason Rhode, Murali Krishnamurthi \\ Northern Illinois University
}

The role of the faculty development center in supporting the academic environment of the institution often includes creating or sustaining a culture of teaching excellence, responding to individual faculty members' needs, and advancing new initiatives in teaching and learning (Sorcinelli, Austin, Eddy, o Beach, 2006). The varied programs, resources, and services offered routinely result from efforts to meet the expressed needs of faculty. While workshops and seminars are effective for introducing new pedagogical approaches or emerging technologies, faculty often have unique questions within specialized contexts that cannot be fully addressed in a large group setting. In such instances, a more personalized and customized level of support is needed. This chapter describes a

\footnotetext{
We express our sincere appreciation to the staff of the Faculty Development and Instructional Design Center at Northern Illinois University for their contributions to and support of this effort. We are especially grateful to program coordinator Brenda Hodges for her coordination of the center's assessment efforts and preparation of assessment and annual reports.
} 
database system for tracking and improving the effectiveness of individual consultation services.

One rewarding and time-consuming form of faculty development is consulting with faculty members on a one-to-one basis. It is through such individualized encounters that support can be tailored to specific expressed needs (Lewis, 2002). Requests for assistance from faculty can consist of a wide variety of formats, ranging from formally scheduled one-on-one meetings to requests by phone, e-mail, or virtual means for technical assistance. During an individualized consultation, faculty development staff have an opportunity to identify and respond to the specific challenge that a faculty member is facing (Kuhlenschmidt, 2010). Consultations regarding a technological challenge present unique opportunities, as the technical discussion often can serve as a platform for examining pedagogical practices and assumptions (Zhu, Kaplan, \& Dershimer, 2011). Such consultations provide a unique way for faculty development staff to offer both instructional design assistance and technology support within the specific context of the faculty member's questions.

\section{Need for Evaluation of Consultations}

It has long been argued and commonly accepted that evaluation is an important component for any successful professional development initiative (Gray \& Shadle, 2009; Kirkpatrick, 1994; Levinson-Rose \& Menges, 1981; Plank \& Kalish, 2010). A comprehensive evaluation strategy yields numerous benefits to a faculty development center, including measuring achievement of mission, vision, and goals (Robertson, 2010); demonstrating impact on teaching and learning (Kucsera \& Svinicki, 2010); and informing decision making and improving practices (Plank \& Kalish, 2010). Centers commonly conduct evaluations to improve programs and services, document successes, and meet reporting mandates (Chism \& Szabó, 1997).

Concerted efforts have been made to understand specifically how faculty development programs evaluate their services. Chism and Szabó (1997) conducted a national survey of centers for teaching and learning (CTLs) and noted that while evaluations of events were frequently performed (over 90 percent of the time), consultations were less frequently and less consistently assessed, due largely to the amount of time needed to record and evaluate them. As a result, they recommended regular efforts 
by institutions to assess user satisfaction in conjunction with more extensive formal studies as resources permit. They advised:

Program staff . . . should be diligent in developing convenient systems for recording who their users are and for assessing satisfaction routinely. Such systems may involve only regular and standard recordkeeping and survey research, which can be analyzed easily with simple database and spreadsheet applications. (p. 61)

Hines (2009) expanded on the comprehensive research by Chism and Szabó (1997) and investigated assessment practices of faculty developers to understand current assessment practices and offer recommendations for improvement. Hines found a consistent lack of evaluation of consultation services offered by faculty development centers. When asked about the lack of routine evaluation of consultation services, respondents noted that issues of confidentiality or service magnitude generally deterred evaluation. Consultation evaluation was believed to leave a paper trail, potentially exposing faculty inadequacies that could result in liability issues. Consultations were also seen as a small, low-impact service not justifying the need to be evaluated.

Hines (2010) extended her 2009 study and investigated assessment methods to measure outcomes of faculty development services at thirtythree established, centralized CTLs across the United States. While efforts at evaluating frequency and impact of many programs and services offered were common, a gap in program evaluation regarding consultation services was again identified. She reported:

Consultation services were not routinely evaluated due to the desire to maintain confidentiality and also due to the perceived lack of time and resources. A small number believed consultation evaluation to be too difficult or the services were too irregular to justify assessing. Others chose not to since evaluations of consultations were not required. (p. 11)

In reality, consultation evaluation can be yet another method for program improvement, fostering the recognition of weaknesses, closing loops, and continual improvement. However, without an electronic system for capturing and analyzing this information, these telling data capable of yielding eye-opening insights that can help inform future strategic efforts are lost. As noted in the literature, the arduous mechanics for using a paper-based process to manually track consultations offered by numerous support staff has prevented the evaluation of consultation services. Implementing an easy-to-use electronic system for capturing 
these data and performing data analysis is the first step to incorporating consultation evaluation as part of a comprehensive assessment effort.

To evaluate better the breadth and scope of consultation services offered to faculty, teaching staff, administrators, and graduate teaching assistants at Northern Illinois University (NIU), the Faculty Development and Instructional Design Center (FDIDC) has implemented a comprehensive consultations tracking system for not only recording consultations but also conducting detailed analysis of consultation services offered, and it uses the results for continuous improvement purposes. The system, implemented as a Microsoft Access database, is one model for recording and analyzing faculty development consultations and can be customized to other universities.

\section{Consultations Data Management}

Consultations are one of the many services FDIDC offers. After attending a particular program, many faculty, teaching staff, administrators, and graduate teaching assistants request individual consultations from center staff to receive more in-depth help on specific needs related to their teaching, technology integration into teaching, or other activities. For example, in 2011-2012, FDIDC offered 1,097 consultations to 398 unique individuals from 88 academic and support units at NIU and a few from outside NIU. The duration of consultations varies from a few minutes to several hours, and the mode of consultations can be face-toface, phone, e-mail, or virtual. We recognized early on the value of information on our consultation services on our planning efforts and decided to operationalize the process for capturing and evaluating the increasing number of consultations offered.

\section{Challenges}

As we initially set out to begin assessing our consultations services, we faced numerous challenges in the manual process for recording and analyzing consultations data, and that led us to implement a standardized consultation evaluation system. Due to the difficulty in distinguishing between various categories of consultations services, we developed formal definitions for each of these categories for the purpose of collecting data that continue to be used. After analyzing past consultations and discussing our data collection and analysis needs for consultations, we defined consultations to be at least thirty minutes in length and require back-and-forth conversation through various means, such as face-to-face meetings or a phone call. Requests (for information) and referrals 
(to other units on campus) are shorter (under thirty minutes) and conducted through the same means as consultations.

Once we agreed on the definitions for the types of consultation services, our staff began individually tracking and categorizing the consultations, requests, and referrals offered each month. In the absence of a formalized system, they selected whatever method for tracking they individually preferred. Some tried electronic spreadsheets for recording consultations data, while others resorted to keeping a note pad at their desk and writing down by hand the date, duration of the consultation, and name of faculty who received the service. The lack of one standardized approach resulted in a simple tally of the number of consultations, requests, and referrals offered. Each staff member compiled the total number of consultations, requests, and referrals offered each month and submitted them to the program coordinator for compiling and reporting. We documented in our annual reports numbers on total consultation services of the various types we offered, but little beyond reporting the frequency of total consultations was possible.

Without a centralized system, staff members were manually keeping track of their consultations individually and were not able to identify accurately the total number of unique consultees they served. For example, while a consultee might be considered "unique" for a particular staff member, that consultee might consult with other staff during the same or a different time period and would not be a truly "unique" consultee for those subsequent consultations. This required considerable time and effort at the end of the reporting period to identify truly new or unique consultees served by our center.

We recognized many obvious limitations to these early efforts at manual tracking of consultation services. Because the data we were attempting to collect were limited to the numbers of total consultations, we were not able to conduct a thorough analysis of our services. Beyond merely tracking the numbers of each type of consultation service, we wanted to collect data on consultation mode, topic, classification and affiliation of consultee, and other useful details. Envisioning the potential for analyzing the consultations offered and using the results to improve our consultation services as well as operational productivity, we realized the need to track various pieces of information associated with each consultation. In addition, we wanted our staff to be freed up from the time-consuming task of tallying and reporting their consultations each month but focus their efforts on offering programs and consultation services. Similarly, we wanted to simplify and speed up the consultation data analysis and assessment process. 


\section{Characteristics of a Tracking System}

In 2009, we conducted a needs analysis to review all aspects of our consultation services and came to the realization that we needed a centralized database system to track our consultation services and resolve the challenges we have been facing in the data collection and analysis process. We identified the following needs for such a consultation tracking system:

1. Data definitions. The system should allow defining and tracking consultation types (consultations, requests, and referrals), modes (face-to-face, e-mail, phone, virtual), consultation topics, consultees details, consulting staff, date and time of each consultation, and any additional open-ended details.

2. Data entry. The data entry process should be simple, consistent, menu driven, and easy for a nontechnical staff person to follow and use. The process should prevent manual look-up of data such as a consultee's department name or classification and allow easy verification that a consultee's information already exists in the system.

3. Data extraction. The data extraction process should be simple and guided by menus and prompts. It should allow a variety of queries for extracting data between date ranges, by particular values, data on a specific consultation or consultee or all consultations or consultees, and enable exporting data easily for analysis or report generation purposes.

4. Data analysis. The system should allow simple analysis of consultations data such as total number of consultations and number of unique consultees and consulting units during a particular period. It should also display required summary data in the form of charts and graphs for analysis purposes at any time by any of staff or the director.

5. Centralized access. The database system should be centrally located on a file server and accessed by all our staff from their work stations through our local area network.

6. Flexibility. The system should be simple and flexible to make changes in the future, and our staff or graduate assistants should be capable of maintaining the system on an ongoing basis.

7. Backup and security. The consultation data should allow easy backup by one person, and not all staff, on a regular basis, and the data stored in system should not be accessible by anyone outside our center. 
8. Affordability. The database software cost should be minimal and not require special licenses, and the system design and development costs should be minimal because our center's budget is very limited.

These needs guided our consultations database system design, development, and implementation process. We recognized that these needs have to be met by the implemented system to be fully functional and usable for tracking our consultations and improving our consultation services.

\section{Consultations Database System Implementation}

To meet the unique needs and requirements of our consultation data management, we internally designed and developed our consultations tracking database system. The design process included traditional systems analysis and design techniques (Kendall \& Kendall, 2008) such as needs analysis, process modeling, and data modeling, as well as relational database system techniques (Silberschatz, Korth, \& Sudarshan, 1999), such as preliminary database design (translation of the data model into an initial database design) and data normalization (elimination of insertion, deletion, and modification data anomalies in the database design) to arrive at a validated final design (Dutka \& Hanson, 1989).

The final design had four tables-for consultee, consultations, staff, and units-that were related to each other through appropriate relationships between them and primary keys that uniquely identified records in each entity table. After considerable analysis of data to be queried or generated as reports from the consultations database system, we defined the necessary columns of each table to capture the data and the rules to enforce the range of acceptable values for those columns. Figure 12.1

\section{Figure 12.1 Data Model of the Consultations Database System}

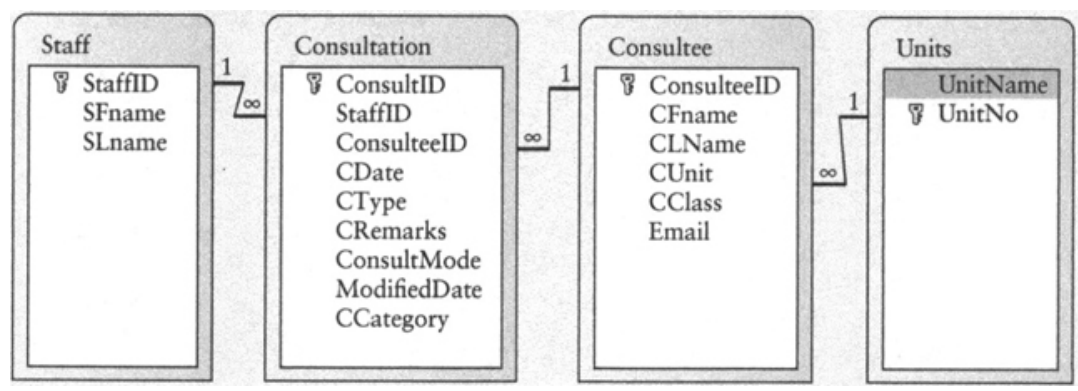


shows the relational data model with the tables, attributes, and relationships between the tables.

\section{System Implementation}

For implementing the design, we chose Microsoft Access due to its affordability (Access is already available on all our staff work stations as part of the Microsoft Office package with no additional cost or licensing necessary), ease of use, and, most important, ease of development, implementation, and maintenance. One of the advantages of Microsoft Access is its graphical user interface, which makes designing queries, reports, and menus easy. Because no programming is required, any of our graduate assistants could also make changes to the system and maintain it in the long run.

Based on how our staff would use the consultations database system, we developed and implemented the user interface menus, data entry forms, queries, and reports using the built-in form, query, and report design features in Microsoft Access. The main menu includes the most used function: Enter Consultee/Consultations; other functions for adding or deleting consultees, consultations, staff, and consultee's units; and a second-level menu for queries and reports. The Queries and Reports menu offers fourteen query and report functions, and a third-level menu with queries for displaying consultation data in the form of nine different charts. Figure 12.2 shows the consultations database system's black-andwhite versions of the main menu and the second-level menu for queries and reports.

Figure 12.2 Screen Captures of the Main Menu (Left) and the SecondLevel Query Menu (Right) of the Consultations Database System
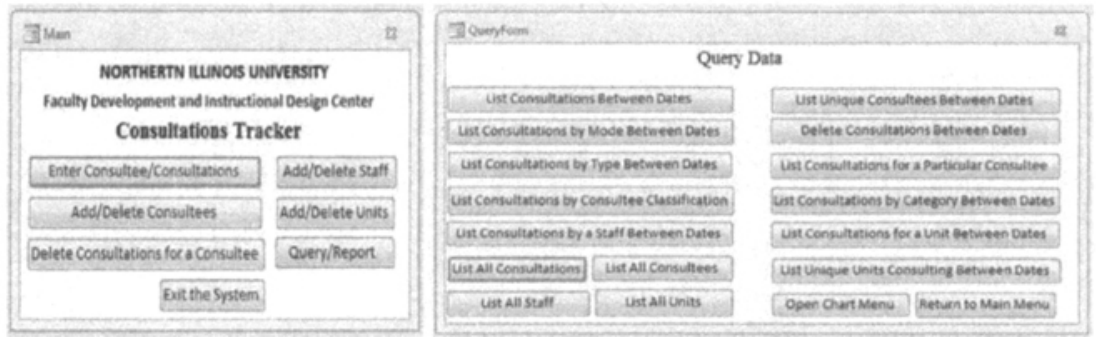


\section{System Features}

When analyzing our staff needs for using the system easily, we recognized that some functions of the system had to be combined for ease of use but also needed the flexibility to be performed independently. For example, our staff wanted to create a new consultee as well as enter consultation data for that consultee on the same screen, but they also wanted the flexibility to create consultees separately as needed without having to enter consultations data. On the data entry screens, we included many drop-down boxes to easily select information such as consultee's employment classification and department, consultation type and mode, consulting staff's name, and date so that our staff do not have to remember such data or spend time looking up the information to enter it. On the data entry screens, our staff also wanted features for searching the database system to see if a consultee already exists in the system to prevent duplicate entries or to locate a consultee quickly to modify his or her information in the system. Figure 12.3 shows the black-and-white versions of the consultation and consultee data entry forms captured from the database system.

One of the important features of the system is the ability for several of our staff to enter their consultations data from their workstations into the same centralized database. For this purpose, the consultations database implemented in Microsoft Access was split into a back-end database containing data definitions and data and a front-end application system containing menus, data entry forms, queries, and reports. We installed the back-end database on a local file server connected to staff work stations

\section{Figure 12.3 Screen Captures of Consultation Data Entry Form and Consultee/Consultation Data Entry Form}
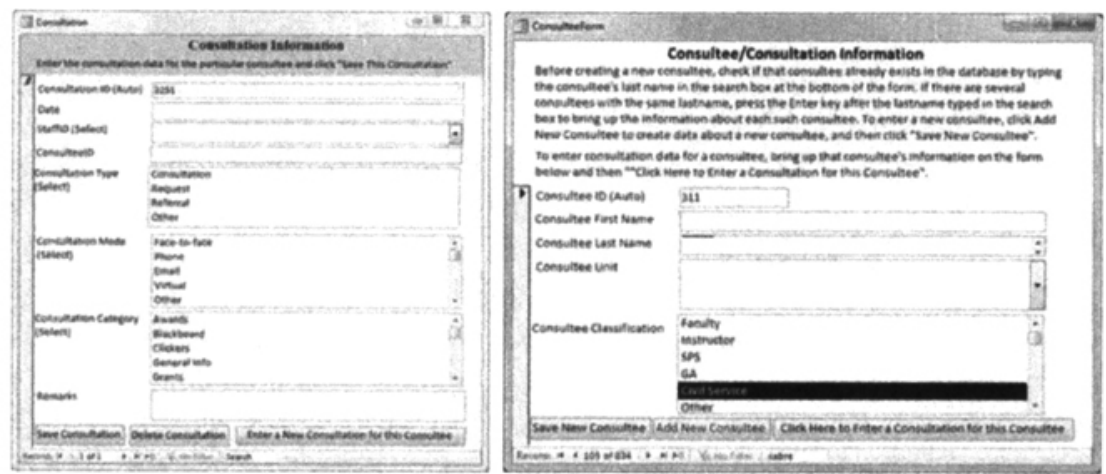
and installed the front-end database application on all staff work stations. This allowed our staff to use the application system on their work stations to access the back-end database independently and store, retrieve, or modify data on the centralized back-end database. Separating the backend database containing the data has also made it easy for regularly backing up only the data instead of unnecessarily backing up the application system as well since it remains the same. Because the database resides on our local file server and not on a web server, the system is secure and cannot be accessed by anyone outside our center.

\section{Database System Use}

The consultation database system has been in use since 2009 at our center, and all staff members use it for entering and querying consultations data. The director and program coordinator use the system for extracting and analyzing consultations data for assessment, planning, and reporting.

\section{Data Entry and Extraction}

The data entry process in the consultation database system is straightforward, and the user interface menus and the data entry screens guide the process. After launching the front-end database application on their workstation, staff members can click the Enter Consultee/Consultations button on the main menu, which will display the Consultee/Consultation Information window, as shown in figure 12.3. In the search box at the bottom of the window, the first or last name of a consultee can be entered to check if the consultee already exists in the system; if the consultee is already in the system, his or her information is displayed on the window, and the staff member can click on Click Here to Enter a New Consultation for This Consultee. Otherwise the staff member can click the Add New Consultee button, enter the new consultee's information, and click the Save New Consultee button to save the data. Then the Click Here to Enter a New Consultation for This Consultee button can be clicked to enter consultations data for that consultee, which will bring up the Consultation Information data entry screen.

On the Consultation Information data entry screen, standardized response types are presented wherever possible as drop-down menu options to save time, prevent mistakes, and compile data in a uniform fashion to aid in data analysis. The data entry fields include the date of the consultation (which can be selected from the calendar displayed), ID of 
staff who offered the consultation, consultation type, consultation mode, consultation category, and remarks. A unique consultee ID is created automatically by the system when a new consultee's data are entered into the system, and, similarly, a unique consultation ID is created by the system when new consultation data are entered into the system and associated with that consultee. After entering consultation details, clicking the Save Consultation button saves the new entry, while the Enter a New Consultation for This Consultee button will save the new entry and display the consultation information screen for entering another consultation data entry for the same consultee. The Delete Consultation button will prevent entered consultation data from being saved.

Once data have been entered into the system, they can just as easily be extracted for analysis and reporting. Currently there are fourteen queries available within the system to view data for specified date range, consultation types, modes, categories, and consulting staff. The data, after being queried from the system, can easily be exported to another application for further analysis or reporting. Staff members can query the system at any time and check the consultations they offered, and the program coordinator can run the queries for assessment and analysis purposes. The chart menu allows the display of queried data in the form of bar or pie charts, which can be used for analysis.

\section{Data Analysis}

The queries and charts available in the consultation system have been helpful for analyzing the consultation data and conduct formative as well as summative evaluation of our consultation services. The data analysis and evaluation efforts have benefited our center in numerous ways:

o Programming. Data analysis on consultations services offered has frequently guided decisions on the need for new programs. If a number of faculty and teaching staff request consultations on the same topic, center staff develop and offer a new program or service to address the unique new needs expressed. For example, in 20102011, more faculty and teaching staff requested consultations than in other years due to a major upgrade of the Blackboard learning management system that year, which introduced many new features. As a result of the increasing number of consultations, center staff offered new programs on those topics, which has reduced the need for consultations on those same topics in 2011-2012.

o Staffing. Analysis of consultation trends over a period of time has helped us to identify peak demand times during the year for 
consultations on particular topics and reassign staff responsibilities to address the demand during those times. Individual staff members have been able to query the system to extract data on their consultations for monthly reporting purposes, and the director has been able to query data on consultations offered by all staff, and use the information for balancing staff workload. Figure 12.4 shows a black-and-white sample of a consultations trend chart extracted from the system.

The consultation system has not only simplified data entry, extraction, analysis, assessment, and reporting but has also improved our staff productivity and consultation services. As a result of the center's assessment efforts, the center was recognized as Outstanding Practices in Assessment by Northern Illinois University's Assessment Panel in February 2012.

\section{Conclusion}

Through our experience collecting, tracking, and analyzing consultations data now for several years, we have found that consultation evaluation can be yet another method for improving programs, recognizing limitations, closing feedback loops, and planning for continuous improvement. Tracking the number, type, mode, and frequency of consultations and consultees' data has provided valuable information for program planning, staffing, and improving support for faculty (see figure 12.5). We now collect, track, and analyze consultations data just as we do for all our other programs and services. Consultation services have become one of our measurable objectives and targets in promoting effective teaching.

Consultations have become a crucial component of services offered by our center and are needed to provide follow-up assistance to faculty after they attend programs or when they could not attend a program due to scheduling conflicts. We have found faculty are usually more enthusiastic about seeking consultations on technology integration, development grants, and career issues, and those consultations have helped our staff address the teaching-related needs of those faculty. As a result of using the consultations database system, our staff are able to provide customized consultation services and follow-up with individual consultees easily.

It is important to remember that any consultations tracking system is only as good as the data entry. Because the data entry cannot be completely automated and requires manual entry into the system by our staff, we continue to look for ways to clarify any questions staff might 


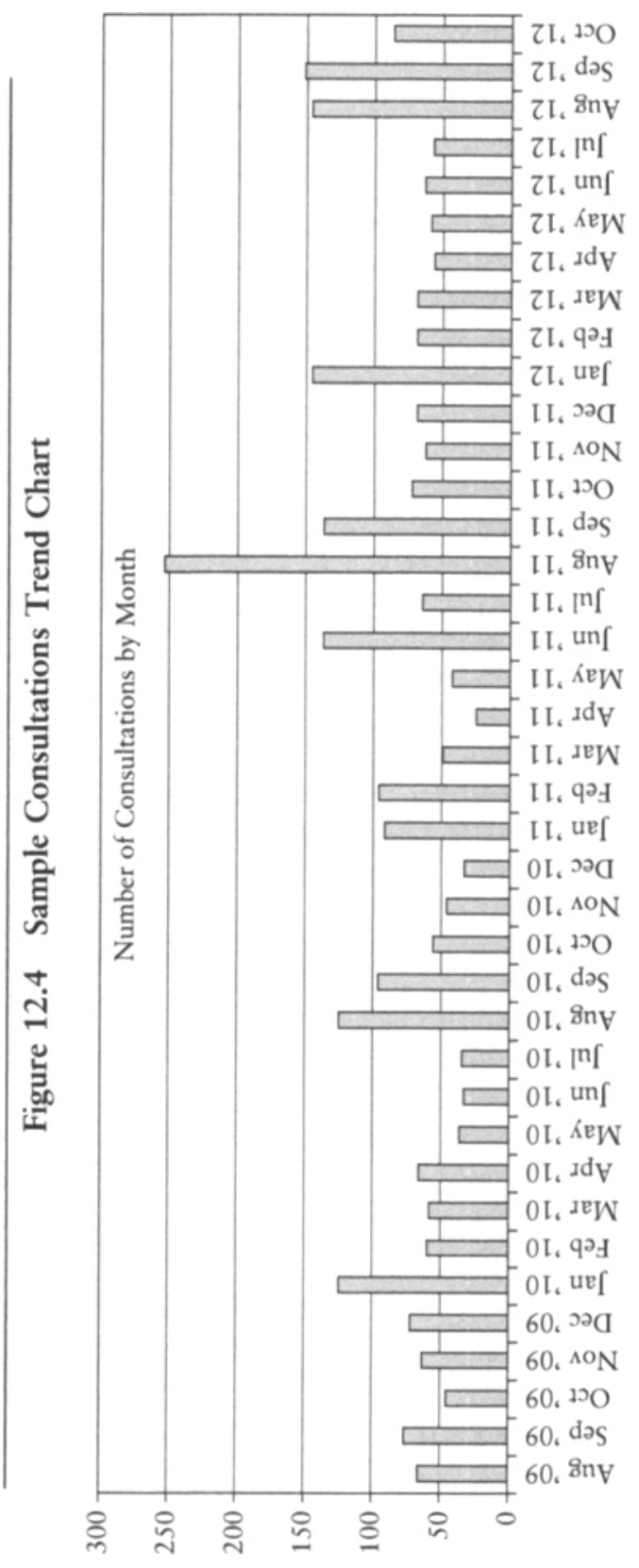


Figure 12.5 Screen Captures of the Chart Menu and a Pie Chart of Consultee Classifications

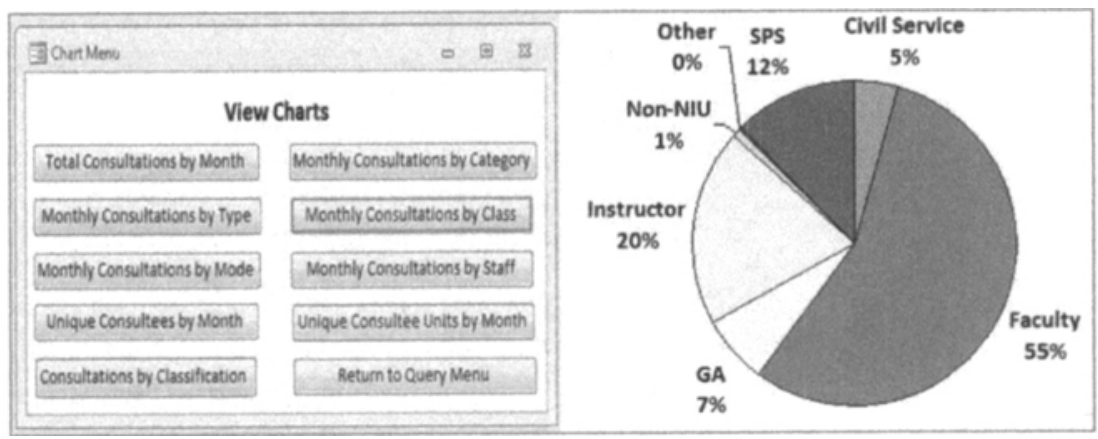

have and encourage best practices for accurate data entry. Staff members have noted that keeping the database open during the day on their computer work station allows quick entry of consultation information immediately after finishing a consultation session. The system has helped individual staff to analyze the consultations they offered, plan their workload, and improve their productivity. Before the consultation database system was implemented, center staff annually spent at least fifty hours to collect and compile manually the consultation data recorded on spreadsheets by eight individual staff and prepare annual reports on consultation services offered by the center. After implementing the consultations database system, this same process takes only a few minutes to generate not only numerical reports but also graphical summaries as needed by any of our staff. Considering that the center director, knowledgeable in database design and development, spent only about forty hours to design, develop, and implement the consultation database system, the system has already paid off within a year, thus justifying the return on the investment of time.

Because we have been using the consultation database system only since 2009 , we plan to explore longitudinal data and trends over more extended time periods to analyze the long-term impact of our consultation services. Over time we will be able to look at multiyear time frames as well as cross-compare frequency, types, and modes with other institutional initiatives and conduct extensive statistical analyses.

Our consultation database system can be used by any faculty development or teaching and learning center and customized for its 
consultation tracking needs or related purposes. Interested faculty development centers can contact our center at facdev@niu.edu for a copy of our consultation database system.

\section{REFERENCES}

Chism, N., \& Szabó, B. (1997). How faculty development programs evaluate their services. Journal of Staff, Program and Organizational Development, 15(2), 55-62.

Dutka, A. F., \& Hanson, H. H. (1989). Fundamentals of data normalization. Los Angeles, CA: Addison-Wesley.

Gray, T., \& Shadle, S. E. (2009). Launching or revitalizing a teaching center: Principles and portraits of practice. Journal of Faculty Development, 23(2), $5-12$.

Hines, S. (2009). Investigating faculty development program assessment practices: What's being done and how can it be improved? Joumal of Faculty Development, 23(3), 5-19.

Hines, S. (2010). An investigation of program assessment practices at established centralized teaching and learning centers: The findings report. Unpublished manuscript, Saint Mary's University of Minnesota.

Kendall, K. E., \& Kendall, J. E. (2008). Systems analysis and design (7th ed.). New York, NY: Prentice Hall.

Kirkpatrick, D. L. (1994). Evaluating training programs: The four levels. San Francisco, CA: Berrett-Koehler.

Kucsera, J. V., \& Svinicki, M. (2010). Rigorous evaluations of faculty development programs. Journal of Faculty Development, 24(2), 5-18.

Kuhlenschmidt, S. (2010). Issues in technology and faculty development. In K. J. Gillespie \& D. L. Robertson (Eds.), A guide to faculty development (2nd ed., pp. 259-274). San Francisco, CA: Jossey-Bass.

Levinson-Rose, J., \& Menges, R. J. (1981). Improving college teaching: A critical review of research. Review of Educational Research, 51(3), 403-434.

Lewis, K. G. (2002). The process of individual consultation. In K. H. Gillespie, L. R. Hilsen, \& E. C. Wadsworth (Eds.), A guide to faculty development: Practical advice, examples, and resources (pp. 53-73). Bolton, MA: Anker.

Plank, K. M., \& Kalish, A. (2010). Program assessment for faculty development. In K. J. Gillespie \& D. L. Robertson (Eds.), A guide to faculty development (2nd ed., pp. 135-149). San Francisco, CA: Jossey-Bass.

Robertson, D. L. (2010). Establishing an educational development program. In K. J. Gillespie \& D. L. Robertson (Eds.), A guide to faculty development (2nd ed., pp. 35-52). San Francisco, CA: Jossey-Bass. 
Silberschatz, A., Korth, H. F., \& Sudarshan, S. (1999). Database system concepts. New York: McGraw-Hill.

Sorcinelli, M. D., Austin, A. E., Eddy, P. L., \& Beach, A. L. (2006). Creating the future of faculty development: Learning from the past, understanding the present. Bolton, MA: Anker.

Zhu, E., Kaplan, M., \& Dershimer, C. (2011). Engaging faculty in effective use of instructional technology. In C. E. Cook \& M. Kaplan (Eds.), Advancing the culture of teaching on campus: How a teaching center can make a difference (pp. 151-166). Sterling, VA: Stylus. 